CLINICAL STUDY

\title{
Evaluation of health-related quality of life in patients with Cushing's syndrome with a new questionnaire
}

\author{
S M Webb, X Badia ${ }^{1}$, M J Barahona, A Colao ${ }^{2}$, C J Strasburger ${ }^{3}$, A Tabarin ${ }^{4}$, M O van Aken ${ }^{5}$, R Pivonello ${ }^{2}$, G Stalla $^{6}$, \\ S W J Lamberts ${ }^{5}$ and J E Glusman ${ }^{7}$ \\ Department of Endocrinology, Hospital Sant Pau, Centre for Biomedical Research on Rare Diseases (CIBERER Unit 747), Autonomous University of \\ Barcelona, Pare Claret 167, 08025 Barcelona, Spain, ${ }^{1}$ Health Economics and Outcomes Research, IMS Health, Centre for Biomedical Research on Rare \\ Diseases (CIBERER Unit 747), 08034 Barcelona, Spain, ${ }^{2}$ Department of Molecular and Clinical Endocrinology and Oncology, 'Federico II' University, \\ 80131 Naples, Italy, ${ }^{3}$ Department of Endocrinology, Charité-Universitätsmedizin, Campus Mitte, 10117 Berlin, Germany, ${ }^{4}$ Department of \\ Endocrinology, Centre Hospitalier Universitaire de Bordeaux, 33600 Pessac, France, ${ }^{5}$ Department of Internal Medicine, Erasmus MC, 3000DR \\ Rotterdam, The Netherlands, ${ }^{6}$ Department of Endocrinology, Max-Planck-Institute of Psychiatry, 80804 Munich, Germany and ${ }^{7}$ Oncology Clinical \\ Research, Novartis Pharmaceuticals Corporation, East Hanover, 07936-1080 New Jersey, USA
}

(Correspondence should be addressed to S M Webb; Email: swebb@santpau.es)

\begin{abstract}
Chronic exposure to hypercortisolism has significant impact on patient's health and health-related quality of life (HRQoL), as demonstrated with generic questionnaires. We have developed a diseasegenerated questionnaire to evaluate HRQoL in patients with Cushing's syndrome (CS; CushingQoL). Objective: Validate the CushingQoL questionnaire in patients with CS in clinical practice conditions. Design: Observational, international, cross-sectional study.

Methods: A total of 125 patients were recruited by 14 investigators from Spain, France, Germany, The Netherlands, and Italy over a 2-month period. Clinical and hormonal data were collected and correlated with results of the generic short form 36 (SF-36) questionnaire, a question on self-perceived general health status and the CushingQoL score.

Results: A total of 107 patients were pituitary-dependent and 18 adrenal-dependent CS; 104 (83\%) were females, mean age 45 years (range 20-73 years); 39 (31\%) were currently hypercortisolemic; and 47 (38\%) adrenal insufficient. In clinical practice, CushingQoL was feasible (117; 94\% of patients fully responded to the questionnaire in a mean time of $4 \mathrm{~min}$ ), reliable (Crohnbach's $\alpha=0.87$ ), and valid (factorial analysis demonstrated unidimensionality and Rasch analysis lead to a final version with 12 items). A significant $(P<0.001)$ correlation was observed between CushingQoL score and patients self-perceived general health status and dimensions of SF-36 (Pearson's correlation coefficient $\geq 0.597$ ). Patients with current hypercortisolism scored worse (lower) than those without $(44 \pm 22$ vs $56 \pm 21, P=0.004)$. Linear regression analysis identified female gender and hypercortisolism as significant predictors for worse QoL. Conclusion: CushingQoL is useful to evaluate HRQoL in patients with CS and correlates with clinical parameters.
\end{abstract}

European Journal of Endocrinology 158 623-630

\section{Introduction}

Chronic exposure to hypercortisolism determines central obesity, gonadal dysfunction, hirsutism, facial plethora, delayed wound healing, muscle weakness, hypertension, hyperglycemia, osteoporosis, and depression, which impact on health-related quality of life (HRQoL) - physically, mentally, and emotionally (1-4). Patients most often complain of fatigue/weakness $(85 \%)$, changes in physical appearance $(63 \%)$, emotional instability (61\%), cognitive problems $(49 \%)$, depression (32\%), and sleeping difficulties (12\%); $80 \%$ report interference with family life and relations with their partner and 56\% with school/work performance (5).

Despite successful treatment of Cushing's syndrome (CS), long-term residual effect on the HRQoL has been observed, which includes poorer physical and social functioning, role limitations due to physical and emotional problems, more pain, and less general wellbeing (2, 6-10). CS patients were those with worst health 6 months after surgery for pituitary adenomas, with more emotional problems (depression and anxiety) and slower recovery than other patients $(11,12)$. Complex pharmacological treatments requiring frequent medical check-ups, and concerns on future health deterioration due to comorbidity, also negatively impact these patients' $\operatorname{HRQoL}(2,13)$.

Clinical recovery is slower than biochemical recovery from hypercortisolism in CS (14). The severity of hypercortisolism and time elapsed since cure does not appear to correlate with QoL (2), but time elapsed since treatment was found to contribute in the physical 
functioning dimension (8). Thus, to optimize control, it seems important to investigate the patient's point of view, and not only objective measures of their illness.

In the absence of a specific questionnaire to evaluate HRQoL in CS, generic health questionnaires - short form 36 (SF-36) or visual analog scale - have been used, together with specific evaluations of cognitive functioning or depression $(6,8,15,16)$. A number of reliable and consistent questionnaires - covering all health dimensions - such as the Hospital Anxiety and Depression Scale (HADS), the General Health Questionnaire-28, the WHO quality of life questionnaireBREF, and the social adjustment scale (SAS1 and SAS2) have been used $(2,13)$.

The high impact of CS on patient's HRQoL led us to develop a specific questionnaire for CS, which would reflect those aspects of greatest concern for the patients, with methodology previously described $(17,18)$. Translations and cultural adaptations were produced as previously described (20) from the initial Spanish version into German, Italian, French, and Dutch, and later to 11 further languages (English, Danish, Polish, Norwegian, Finnish, Turkish, Flemish, Greek, Bulgarian, Mandarin Chinese, and Portuguese - with an additional cultural adaptation for Brazil; further cultural adaptations were also performed for Argentinean Spanish, for Belgian and Canadian French, as well as for USA and Canadian English). Each translation was presented to five patients whose native language was that of the translated version, to assess and correct comprehension, clarity, cultural relevance, and suitable wording (cognitive debriefing), providing the final translated version.

The aim of this study was to evaluate the psychometric properties (validity and reliability) of this diseasegenerated HRQoL questionnaire (CushingQoL) and its correlation with clinical parameters relevant to patients with CS.

\section{Patient and methods}

\section{Patients}

To calculate the sample size, and assuming moderate (i.e., between 0.3 and 0.7 ) correlation coefficients between the scores of the CushingQoL and SF-36 questionnaires and $10 \%$ of incomplete answers, with a level of significance of 0.05 and a statistical power of 0.80 , a minimal sample of 76 patients was required.

One hundred and twenty-five patients aged 18 years or above with histologically proved CS of pituitary or adrenal origin, or whose hypercortisolism disappeared after adrenal or pituitary surgery were included in this observational, international, multicenter, and crosssectional study. In newly diagnosed patients, CS was considered confirmed if hypercortisolism was not suppressible by low dose $(1 \mathrm{mg})$ dexamethasone in the presence of elevated urinary free cortisol. All participants gave written informed consent to participate after local ethics committee approval. Patients with CS due to adrenal carcinoma, ectopic adrenocorticotrophin (ACTH) syndrome, or exogenous treatment with glucocorticoids were excluded.

Since CS is a rare disease with a low incidence, participation of 14 investigators from five European countries (Spain, France, Germany, the Netherlands, and Italy) was necessary to achieve the required sample of patients over a 2-month period (between August and October 2006). At a single visit, data from the medical records and response to HRQOL questionnaires were collected. Sociodemographic data (age, gender, level of studies, and current employment status) and the following clinical variables were collected: weight, height, blood pressure, date of diagnosis of CS and cause (pituitary or adrenal adenoma), history and persistence or not of adrenal insufficiency and hypercortisolism, surgery undergone for the disease (type, date, route, and results of histology), and history, dose, and date of pituitary radiotherapy. Recent $24 \mathrm{~h}$ free urinary cortisol (FUC, within the last 6 months) and details on current pharmacological treatment (adrenolytic, dopaminergic, or hydrocortisone substitution), concomitant diseases and their treatment, and hospital admissions over the last year related to CS or its complications were recorded. Thus, a wide array of clinical situations which CS patients can perceive during the course of their illness, ranging from severe hypercortisolemia to post-therapy adrenal insufficiency were included; the rationale for this was that the emergent questionnaire would be applicable in all these patients. Hormones were measured locally with commercial kits for which normal reference values were available; each investigator reported whether the values were high, low, or within the normal range. Inter- and intrassay of variation of the assays were all $<10 \%$. Plasma ACTH in $\mathrm{pg} / \mathrm{ml}$ were transformed into $\mathrm{pmol} / \mathrm{l}$ (by multiplying 0.22).

\section{HRQoL evaluation}

The CushingQoL questionnaire was developed following standard methodology (18); the clinical characteristics of the ten patients interviewed in order to identify HRQoL domains and clinical aspects of the disease are described in Table 1. Interviews took place in an office and patients were asked by an endocrinologist, specifically trained to perform these interviews, to describe how CS had affected their lives; if they did not spontaneously mention them, they were asked about the domains previously identified; interviews were taped and later transcribed.

The CushinQoL questionnaire with a time frame referred to the preceding 4 weeks, was presented to the patients in an office by a nurse or physician after explaining the reasons, and ensuring correct understanding after reading the instructions (Table 2). 
Table 1 Clinical characteristics of the patients interviewed to identify the items important for health-related quality of life (HRQoL) in Cushing's syndrome.

\begin{tabular}{lccccc}
\hline Patient & Gender & Age & $\begin{array}{c}\text { Active } \\
\text { hypercor- } \\
\text { tisolism }\end{array}$ & Diagnosis & $\begin{array}{c}\text { Pituitary } \\
\text { radiotherapy }\end{array}$ \\
\hline 1 & M & 41 & Yes & CD & Yes \\
2 & F & 63 & Yes & CD & Yes \\
3 & F & 36 & Yes & CD & Yes \\
4 & M & 32 & No & CD & No \\
5 & M & 63 & No & CD & No \\
6 & F & 63 & No & Adrenal & No \\
7 & F & 36 & No & Adrenal & No \\
8 & F & 46 & No & CD & No \\
9 & F & 46 & Yes & CD & Yes \\
10 & F & 36 & Yes & CD & Yes \\
\hline
\end{tabular}

$C D$, pituitary Cushing's disease; $M$, male; $F$, female.

Answers based on Likert scales with five response categories: 'Always', 'Often', 'Sometimes', 'Rarely', and 'Never', or 'Very much', 'Quite a bit', 'Somewhat', 'Very little', and 'Not at all', were rated on a scale of 1-5, where ' 1 ' corresponds to 'Always' or 'Very much' and ' 5 ' to 'Never' or 'Not at all'. Therefore, the lower the score, the greater the impact on HRQoL. The score is the sum of all the item responses and can range from 12 (worst HRQoL) to 60 points (best HRQoL). The score can be interpreted if the number of unanswered items does not exceed 3 (25\% of the questions). To simplify interpretation of scores, standardization on a scale from 0 (worst HRQoL) to 100 (best HRQoL) can be done with the following formula:

$$
Y=\frac{(X)-\min }{(\max -\min )} \times 100
$$

where $Y$ is the recalculated score, $X$ is the sum of all the item responses within the study score, 'min' is the minimum $(\min =12)$, and ' $m a x$ ' is the maximum possible score $(\max =60)$.

Answers to the generic SF-36 questionnaire (19) and a question on self-perceived general health status were also collected. The SF-36 questionnaire previously used in patients with CS or acromegaly $(2,6,8,15,16)$ contains 36 items, which covers eight dimensions and provides an overall measure of general health and specific dimensions of vitality, physical and social functioning, functional and emotional performance, pain, effects on work, and mental health. High scores indicate good health status. Patients were also asked to assess their self-perceived health status through seven possible ratings to the question 'Mark the answer which best defines your health today in relation to your CS'(from very bad, fairly bad, slightly bad, through neither good nor bad to slightly, fairly, or very good).

\section{Statistical analysis}

Data were analyzed using the SPSS statistical package version 14.0 for Windows (SPSS, Chicago, IL, USA). A statistical level of significance of 0.05 was used. A descriptive analysis was made of sociodemographic and clinical characteristics (age, gender, disease duration, diagnosis, surgery, hormone values, pharmacological treatment, and concomitant illnesses) using mean and S.D. for continuous variables, and number and percentage of patients by response category for the categorical variables. Correlations of the CushingQoL scores with the SF-36 questionnaire and general perceived health were analyzed with a Pearson's correlation coefficient. Distribution of data was analyzed by the Kolmogorov-Smirnov's test; normally distributed data were compared using a Student's $t$-test and nonnormally distributed data with a Mann-Whitney $U$ test.

For validation of the CushingQoL questionnaire, an exploratory factorial analysis was performed to determine the dimensions, followed by a Rasch analysis, a powerful model belonging to the item response theory, based on the idea that some items are more important than others, and should be ordered hierarchically depending on the importance attributed to them by patients (21). Initial calibration was developed for each of the initial 34 items, followed by an evaluation of how

Table 2 Items included in the CushingQoL questionnaire.

1. I have trouble sleeping (I wake up during the night; it takes me a long time to get to sleep, etc.)

2. I have pain that keeps me from leading a normal life

3. My wounds take a long time to heal

4. I bruise easily

5. I am more irritable, I have sudden mood swings and angry outbursts

6. I have less self-confidence, I feel more insecure

7. I'm worried about the changes in my physical appearance due to my illness

8. I feel less like going out or seeing relatives or friends

9. I have had to give up my social or leisure activities due to my illness

10. My illness affects my everyday activities such as working or studying

11. It's difficult for me to remember things

12. I'm worried about my health in the future

The instructions read: the following sentences refer to what you may think or feel about your Cushing's syndrome. Your answers will help us to know how you feel and how much your illness has interfered in your usual activities in the past 4 weeks. Below each sentence you will find several response choices. Please read each sentence carefully. After reading each sentence, check the box next to the answer that best describes what you think is happening to you. There are no rights or wrong answers. We are simply interested in what is happening to you because of your Cushing's syndrome. 
each item contributed to the overall measurement of health; redundant items were eliminated. After individual initial analysis of each dimension, the CushingQoL questionnaire demonstrated to be unidimensional (involving sub-components referent to daily life, emotional, and physical aspects domains) with a final content of 12 questions (Table 2).

In order to relate CushingQoL scores and other variables, a linear regression model was used, using as independent variables, sociodemographic (age and gender) and clinical characteristics (age at diagnosis and FUC levels).

\section{Results}

\section{Clinical data}

One hundred and twenty-five patients with CS were included from Spain, France (four sites each), Germany (three sites), Italy, and the Netherlands (one site each; Table 3). One hundred and four (83\%) were women; $79 \%$ had secondary or university studies, $43 \%$ of patients were employed, $22 \%$ were homemakers, $14 \%$ were retired, and $10 \%$ had temporary or permanent disability. Mean (s.D.) age was 45.3 (13.1) years (range 20-73). Diagnosis was made a mean of 61 months before, ranging from newly diagnosed to 24 years; $6 \%$ were diagnosed in the 1980 s, $20 \%$ in the 1990 s, $55 \%$ between 2000 and 2005, and 19\% were recently diagnosed in 2006.

Eighty-six percent $(n=107)$ were diagnosed with pituitary-dependent CS and 14\% $(n=18)$ cortisolsecreting adrenal adenoma. Mean (s.D.) body mass index (BMI) was $27.8(6) \mathrm{kg} / \mathrm{m}^{2}$, ranging from 17.6 to $45.7 \mathrm{~kg} / \mathrm{m}^{2} ; 31 \%$ of patients were obese (BMI $>30$ ) and 5 with morbid obesity (BMI $>40$ ).

At the study visit, 39 (31\%) of patients were considered to be hypercortisolemic by the reporting endocrinologist (they either had increased $24 \mathrm{~h}$ UFC, or in 12 cases had normal UFC on medical therapy). Of 125 patients, $28(22 \%)$ were receiving pharmacological treatment for CS (ketokonazole in 18, metyrapone in 7 , mitotane in 1 , and cabergoline in 9, alone or in combination). One hundred and five had undergone surgery (91 out of 107 (85\%) pituitarydependent Cushing's disease: transsphenoidal in 90 cases of which 7 also underwent adrenal surgery and 1 bilateral adrenalectomy; 14 out of 18 (78\%) adrenal adenomas had undergone unilateral adrenalectomy); 68 out of the 90 transsphenoidal surgeries disclosed positive immunohistochemistry for ACTH (77\%; in the remaining 22 ACTH-staining was either not available or negative); in adrenal-dependent CS, an adenoma was confirmed histologically in 13 and an oncocytoma in 1.

Fifty-three percent $(n=66)$ developed adrenal insufficiency after treatment for CS, diagnosed a mean of 39 (51) months before the study visit (range 0-280 months); at the study visit, 47 (38\% of the total sample) were still adrenal insufficient, of which 46 were receiving substitution treatment (35 with hydrocortisone, 11 with cortisone acetate; 4 were also on fludrocortisone). In 17, adrenal insufficiency had recovered a mean of 5 years before $(60 \pm 70$ months, range 1-270 months). Mean plasma ACTH was 30.4 (interquartile range 13.6-55.9) pg/ml.

Of the 107 patients with pituitary-dependent CD, 24 $(22 \%)$ underwent pituitary radiotherapy (conventional $38 \%$, radiosurgery $12 \%$, and fractionated stereotactic radiotherapy $50 \%$ ), administered $74 \pm 69$ months before (range 2 months to 24 years).

Of the 125 patients, $100(80 \%)$ had concomitant diseases (hypertension 40, diabetes mellitus 20, osteopenia/osteoporosis 42 , and depression 28) and 42 out of the 107 pituitary-dependent Cushing's disease had hypopituitarism (39\%; 35 growth hormone, 23 thyrotrophin, 18 gonadotrophin, and 11 anti-diuretic hormone deficient). Over the preceding year, 62 (50\%) had been hospitalized in relation to their CS or derived

Table 3 Clinical characteristics of the patients.

\begin{tabular}{|c|c|c|c|c|c|c|}
\hline & Spain & France & Germany & The Netherlands & Italy & Total \\
\hline \multicolumn{7}{|l|}{ Age } \\
\hline Mean & 45.8 & 39.8 & 49.2 & 47.3 & 45.0 & 45.3 \\
\hline S.D. & 12.3 & 13.8 & 13.0 & 13.6 & 12.2 & 13.1 \\
\hline Median & 46.0 & 37.0 & 50.5 & 42.0 & 47.0 & 46.0 \\
\hline Interquartile range & $36.5-57.0$ & $28.7-53.2$ & $42.2-56.5$ & $37.0-59.0$ & $34.5-53.5$ & $35.0-55.0$ \\
\hline \multicolumn{7}{|l|}{ Age at diagnosis } \\
\hline S.D. & 13.2 & 12.7 & 13.3 & 13.4 & 13.2 & 13.6 \\
\hline Median & 38 & 34.5 & 44.0 & 42.0 & 35.0 & 40.0 \\
\hline Interquartile range & 28.5-50.0 & $25.5-45.2$ & $37.0-53.7$ & $36.5-56.0$ & $23.5-45.5$ & $29.0-49.0$ \\
\hline \multicolumn{7}{|c|}{ Time since diagnosis (months) } \\
\hline Mean & 69.1 & 56.1 & 45.0 & 25.0 & 101.5 & 60.9 \\
\hline S.D. & 69.0 & 74.7 & 56.0 & 16.7 & 77.4 & 67.9 \\
\hline Median & 40.0 & 36.0 & 17.5 & 20.0 & 80.0 & 38.0 \\
\hline Interquartile range & $15.5-121.5$ & $3.0-86.5$ & $8.2-62.7$ & $15.5-41$ & $41.5-152.5$ & $11.5-83.5$ \\
\hline
\end{tabular}


complications (of which 53\% were admitted once, $36 \%$ twice, and $11 \%$ thrice).

\section{Evaluation of HRQoL}

With generic questionnaires, self-perceived health status was good (between fairly and very good) in 40 (34\%), slightly good in $22(19 \%)$, neither good nor bad in $24(20 \%)$, slightly bad in $12(10 \%)$, and bad in 19 (16\%; fairly to very bad). Mean (S.D.) scores in each dimension of the SF-36 questionnaire were 65 (27) in physical functioning, 55 (31) in role physical, 58 (32) in bodily pain, 47 (22) in general health, 48 (23) in vitality, 64 (29) in social functioning, 63 (32) in role emotional, and 59 (23) in mental health. No differences were seen when comparing patients with or without elevated FUC, although in the former, values tended to be lower. These results are all lower (worse QoL) then those reported by van Aken et al. in cured CD patients who scored worse than general population for each dimension of the SF-36 questionnaire (2).

With the CushingQoL questionnaire, mean (s.D.) global CushingQoL score was 53 (22); only one patient obtained the highest (best possible HRQoL assessment), while none obtained the lowest score. Table 4 shows the scores globally and in each country. Only one patient exceeded the maximum allowed unanswered questions; therefore, psychometric properties were evaluated with a sample of 124 patients. The Kolmogorov-Smirnov statistic test confirmed normal distribution of data.

Mean time of administration was 4 min and 94\% completed all 12 items. The reliability of CushingQoL was measured by Crohnbach's $\alpha$ coefficient which refers to the extent to which the questions from one scale or dimension measure different aspects of the same concept; this coefficient was 0.87 , above the minimum required for psychometric standards $(0.70)$, reflecting good internal consistency of this unidimensional construct.

CushingQoL scores decreased $(P<0.001)$, as selfperceived health status worsened (Fig. 1), detecting a decrease $>50$ points between patients with very good and very bad health status. CushingQoL scores were moderately correlated ( $r$ between 0.3 and 0.7 ) with all dimensions of the SF-36 questionnaire (Pearson's correlation coefficient: physical 0.670 , role physical
0.708 , bodily pain 0.602 , general health 0.597 , vitality 0.716 , social functioning 0.676 , role emotional 0.638 , and mental health 0.706).

Patients diagnosed in the last 2 years $(n=50)$ scored worse than those diagnosed more than 2 years before $(n=74, P<0.001$; Table 5). Current hypercortisolism (i.e., reported by the endocrinologist with concomitant elevation of 24-h FUC, $n=26$ ) was associated with worse scores in the CushingQoL questionnaire than patients without $(n=60, P<0.004)$. The same was true when only the patients with pituitary-dependent CS were analyzed: $46 \pm 21$ in active CS versus $57 \pm 21$ in nonactive, $P=0.013)$. No correlation was observed between time elapsed since surgery and the CushingQoL score.

Admission to hospital over the prior year resulted in lower scores in the CushingQoL questionnaire $(n=61$, $47 \pm 22)$ than no admissions $(n=63,59 \pm 21$, $P=0.002$; Table 5). No relation was identified between the CushingQoL score and the presence $(n=44,56 \pm$ $23)$ or not $(n=77,52 \pm 21)$ of adrenal insufficiency; however, time elapsed since diagnosis of adrenal insufficiency was slightly but positively related with the CushingQoL score $(r=0.35, P=0.02)$; in other words, the longer the duration of adrenal insufficiency, the greater the impact on HRQoL.

In pituitary-dependent CS, the presence $(n=40,56 \pm$ 21) or not of hypopituitarism $(n=66,53 \pm 22)$ or prior pituitary radiotherapy (irradiated $n=24,5 \overline{5} \pm 19$, versus non-irradiated $n=81,54 \pm 23$ ) did not determine differences in the CushingQoL score.

The linear regression analysis using as the dependent variable the CushingQoL questionnaire score identified female gender $(P=0.029)$ and elevated FUC $(P=0.011)$ as main contributors to impaired HRQoL, while current age and age at diagnosis were not significantly correlated.

\section{Discussion}

Except for some descriptive studies, no results using disease-specific questionnaires with standardized methodology to evaluate HRQoL were available up to now in patients with CS. A retrospective questionnaire referring to employment status, work capacity, symptoms, perceptions as to being cured, changes in

Table 4 CushingQoL scores in Cushing's syndrome (CS) patients.

\begin{tabular}{|c|c|c|c|c|c|c|}
\hline & Spain & France & Germany & The Netherlands & Italy & Total \\
\hline Mean & 54 & 46 & 57 & 49 & 59 & 53 \\
\hline S.D. & 24 & 18 & 26 & 19 & 20 & 22 \\
\hline Minimum & 15 & 8 & 18 & 15 & 19 & 8 \\
\hline 25th percentile & 33 & 36 & 31 & 32 & 43 & 37 \\
\hline Median & 54 & 46 & 58 & 50 & 58 & 51 \\
\hline 75th percentile & 75 & 55 & 77 & 62 & 76 & 70 \\
\hline Maximum & 100 & 87 & 96 & 90 & 94 & 100 \\
\hline Valid $n$ & 29 & 26 & 23 & 21 & 25 & 124 \\
\hline
\end{tabular}




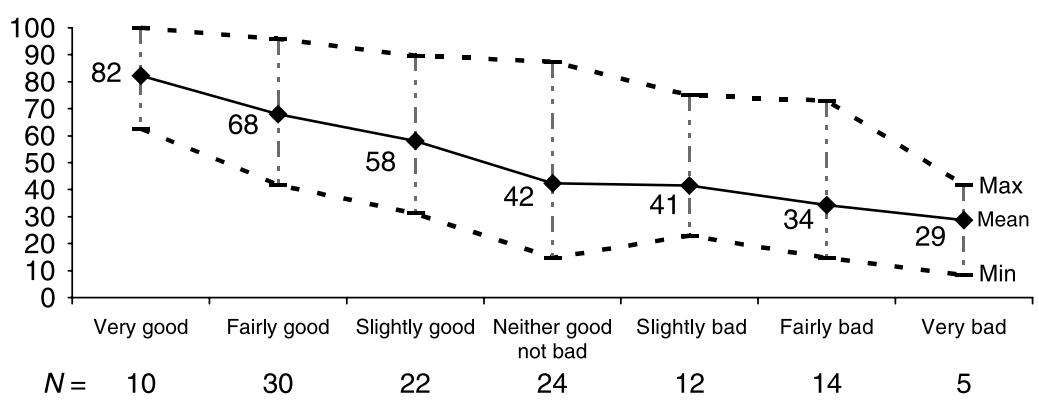

Figure 1 Mean, minimum, and maximum values of the CushingQoL scores in relation to the patients' self-perceived health status. health following treatment, and medication received has been described (14), as well as steroid substitution, working capacity, abnormal pigmentation, and other disorders suffered by the patient (21). The patient survey designed by Gotch included sociodemographic and clinical data (age, year of diagnosis, treatment received), questions on CS effects on patient's life and family, its physical and mental effects, and effects on school/work performance (5).

With this newly developed disease-generated CushingQoL questionnaire, we have evaluated HRQoL in patients with CS. This questionnaire available in 16 languages is brief, easy to complete and has preliminarily demonstrated feasibility and good psychometric properties of validity and reliability. Given the crosssectional nature of this study, test-re-test reliability and sensitivity to change (i.e., demonstrating over time no change in CushingQoL score in patients with stable disease, and in contrast, significant improvement after successful therapy) have yet to be confirmed, although the observation of worse scores in hypercortisolemic patients in comparison to those who were not, is promising. Since the final version is unidimensional, it offers a global score, easier to deal with than if it were multidimensional (i.e., emotional, physical, social, etc).

In this multinational study, CushingQoL scored worse in patients with recent hospital admission and diagnosed in the prior 2 years, when all had experienced hypercortisolism, a situation previously described as associated with impaired perception of $\operatorname{CoL}(1,8,9,15)$. Current hypercortisolism determined a worse score in CushingQoL when compared with patients who were not cushingoid. Using the SF-36 questionnaire, greater impairment of QoL in active CS patients has been observed than in cured patients $(8,15)$, which is however, not associated with normalization of QoL, even after long-term control of hypercortisolism (2, 15); the degree of initial hypercortisolism does not appear to determine subsequent level of impairment of HRQoL either, since initial $24 \mathrm{~h}$ FUC excretion prior to therapy did not correlate with HRQoL (2). In parallel, psychopathology (mainly atypical depression) highly prevalent at baseline (66.7\%) improves 1 year after treatment, but suicidal ideation and panic attacks may increase after control of hypercortisolism (9). Since cognitive function is also impaired by hypercortisolism, especially the declarative memory system, these impairments explain the patients' demoralization and psychological distress after remission of CS (1) and consequently, the great impact on HRQoL.

We observed no correlations between CushingQoL and time elapsed since surgery; the latter was also observed by van Aken (2) in cured patients, where duration of cure did not correlate to any QoL scales; in contrast, we did not observe any influence of hypopituitarism or adrenal insufficiency on the CushingQoL score, while van Aken did (2). They used the SF-36 and Nottingham Health Profile (NHP) questionnaires, as well as multidimensional fatigue index-20 (MFI-20) to assess fatigue and HADS to evaluate anxiety and depression in 58 long-term cured CD patients; reduced scores for all sub-scales were observed compared with normal controls, but associated hypopituitarism was the main independent predictor of reduced HRQoL after successful treatment, despite replacement therapy; other determinants were age, age at diagnosis, gender, and anxiety/depression (2). Nevertheless, hypopituitarism was especially determinant for decreased QoL for

Table 5 Relationship between CushingQoL scores and clinical variables.

\begin{tabular}{|c|c|c|c|}
\hline & & $\begin{array}{l}\text { CushingQoL } \\
\text { score }\end{array}$ & $P$ value \\
\hline Time elapsed since diagnosis & $\begin{array}{l}\text { Recent diagnosis ( } \leq 24 \text { months; } n=50 \text { ) } \\
\text { Old diagnosis ( }>24 \text { months; } n=74)\end{array}$ & $\begin{array}{l}44 \pm 22 \\
59 \pm 20\end{array}$ & $<0.001$ \\
\hline $\begin{array}{l}\text { Clinically, active and concomitant high free } \\
\text { urinary cortisol }\end{array}$ & $\begin{array}{l}\text { No }(n=60) \\
\text { Yes }(n=26)\end{array}$ & $\begin{array}{l}56 \pm 21 \\
44 \pm 22\end{array}$ & 0.004 \\
\hline Hospital admissions & $\begin{array}{l}\text { No admissions }(n=63) \\
\text { With admissions }(n=61)\end{array}$ & $\begin{array}{l}59 \pm 21 \\
47 \pm 22\end{array}$ & 0.002 \\
\hline
\end{tabular}


HADS and MFI-20 where hypopituitary patients were severely impaired, while non-hypopituitary subjects were similar to controls. In NHP and SF-36 scores, influence of hypopituitarism was less pronounced, since both groups of CD patients scored worse than controls on most dimensions, and for SF-36, additional hypopituitarism only determined greater impairment for scores related to emotional role, pain, and general health. It is worth pointing out that in our experience the closest correlation of CushingQoL was with the vitality dimension of the SF-36 (which was left out by van Aken since they used the MFI-20 to evaluate vitality, energy, and fatigue). Our cross-sectional study included both hyper-, normo- and hypocortisolemic patients after treatment; the lack of any difference in score for those CS patients who were hypopituitary would suggest that impairments picked up by the CushingQoL questionnaire are predominantly related to hypercortisolism rather than hormone deficiencies, and that current hypercortisolism is a stronger determinant for patient well-being and HRQoL impairment, so any additional contribution of hypopituitarism may be masked. This is supported by the linear regression analysis in which elevated FUC and female gender were the main contributors to impaired HRQoL.

Pituitary radiotherapy was not associated with worse QoL measured with SF-36 in cured patients (2) or in our experience with the CushingQoL questionnaire either. This contrasts with findings in acromegaly where radiotherapy does determine a worse HRQoL (23), and may be interpreted as meaning that CS itself has a more profound deleterious effect on the patient than acromegaly; in fact, the mean scores for SF-36 reported in these cured Dutch acromegalic patients were all higher (less affected) than those attained by the CS patients reported here, even when only those without current hypercortisolism were separately analyzed, the most affected being general health and vitality. Nevertheless, acromegalic patients were not completely comparable, since their mean age was 14 years older (and age tends to worsen HRQoL), and included more males (52\%), in contrast to the female predominance seen in CS $(85 \%$; and females tend to exhibit worse HRQoL than males).

Lindholm observed no abnormality in perceived health using SF-36 in patients treated for adrenal adenoma, in sharp contrast with pituitary-dependent $\mathrm{CD}$, both cured and active (the latter being worse). We were unable to demonstrate differences in the CushingQoL score in CS of pituitary or adrenal origin, although the small number of the latter makes any comparison difficult.

Patients with worse self-perceived health or score on SF-36, also scored worse in the CushingQoL questionnaire, supporting the view that this new tool is capable of reflecting HRQoL. The advantage of a robust and widely validated generic questionnaire such as the SF-36 both in large series of general population and different patient groups, contrasts with the advantage of identifying those dimensions most important for HRQoL in patients with CS, included in the CushingQoL, which should therefore be more sensitive to pick up clinically relevant changes. Only one-third of patients perceived their health as good and another third as bad. Eighty percent of these patients had concomitant diseases (mostly hypertension, diabetes mellitus, depression, and osteoporosis), confirming that CS patients are a sick population (1). This is also evidenced when SF-36 results obtained in different endocrine diseases such as adrenal insufficiency $(24,25)$, primary hyperparathyroidism (26), thyroid carcinoma (27), craniopharyngioma (28), and subclinical hypothyroidism (29) are compared, since all scored better than the CS cohort reported here but worse than normal population.

The mechanisms through which CS determines HRQoL impairment are probably multifactorial involving physical and psychological features. Severe fatigability and changes in body composition and image, anxiety, irritability, mood swings, depression, decreased memory, less self-confidence, and difficulties in sleeping are common and the main concerns for these patients. Glucocorticoids are known to affect behavior, mood, neural activity, memory, and other processes in the central nervous system $(1,30)$, and to reduce brain volume in a reversible way when hypercortisolism is controlled (31). However, other dimensions such as cognition (4) or body composition (32) do not normalize after endocrine cure, strongly suggesting that these changes are not fully reversible, nor is impaired HRQoL.

In summary, the disease-generated CushingQoL questionnaire is a feasible, reliable, and valid instrument for measuring HRQoL in patients with CS; the short number of items and the unidimensionality contribute to facilitate its scoring and interpretation. Scores correlate with relevant clinical parameters. Sensitivity to change and test-re-test reliability remain to be confirmed in longitudinal studies. Even though these preliminary results are promising, further testing is required to validate the usefulness of this questionnaire.

\section{Acknowledgements}

The authors wish to thank Mrs Pilar Pérez and Àgata Carreño as well as Drs Irene Halperin, Manuel PuigDomingo, Jaume Puig, Isabel Salinas, Mónica Recasens, Wifredo Ricart, Thierry Brue, Jerome Bertherat, Olivier Chabre, and Klaus Mann for their contribution in the development of this project. Supported by an unrestricted grant from Novartis, New Jersey, USA.

\section{References}

1 Arnaldi G, Angeli A, Atkinson AB, Bertagna X, Cavagnini F, Chrousos GP, Fava GA, Findling JW, Gaillard RC, Grossman AB, Kola B, Lacroix A, Mancini T, Mantero F, Newell-Price J, 
Nieman LK, Sonino N, Vance ML, Giustina A \& Boscaro M. Diagnosis and complications of Cushing's syndrome: a Consensus Statement. Journal of Clinical Endocrinology and Metabolism 2003 88 5593-5602.

2 van Aken MO, Pereira AM, Biermasz NR, van Thiel SW, Hoftijzer HC, Smit JW, Roelfsema F, Lamberts SW \& Romijn JA. Quality of life in patients after long-term biochemical cure of Cushing's disease. Journal of Clinical Endocrinology and Metabolism 200590 3279-3286.

3 Sonino N \& Fava GA. Psychosomatic aspects of Cushing's disease. Psychotherapy and Psychosomatics 199867 140-146.

4 Forget H, Lacroix A \& Cohen H. Persistent cognitive impairment following surgical treatment of Cushing's syndrome. Psychoneuroendocrinology 200227 367-383.

5 Gotch PM. Cushing's syndrome from the patient's perspective. Endocrinology and Metabolism Clinics of North America 199423 607-617.

6 Hawn MT, Cook D, Deveney C \& Sheppard BC. Quality of life after laparoscopic bilateral adrenalectomy for Cushing's disease. Surgery 2002132 1064-1068.

7 Kelly WF, Kelly MJ \& Faragher B. A prospective study of psychiatric and psychological aspects of Cushing's syndrome. Clinical Endocrinology $1996 \mathbf{4 5} 715-720$.

8 Lindsay JR, Nansel T, Baid S, Gumowski J \& Nieman LK. Long-term impaired quality of life in Cushing's syndrome despite initial improvement after surgical remission. Journal of Clinical Endocrinology and Metabolism 200691 447-453.

9 Dorn LD, Burgess ES, Friedman TC, Dubbert B, Gold PW \& Chrousos GP. The longitudinal course of psychopathology in Cushing's syndrome after correction of hypercortisolism. Journal of Clinical Endocrinology and Metabolism 199782 912-919.

10 Sonino N, Navarrini C, Ruini C, Ottolini F, Paoletta A, Fallo F, Boscaro M \& Fava GA. Persistent psychological distress in patients treated for endocrine disease. Psychotherapy and Psychosomatics $20047378-83$.

11 Flitsch J, Spitzner S \& Lüdecke DK. Emotional disorders in patients with different types of pituitary adenomas and factors affecting the diagnostic process. Experimental and Clinical Endocrinology and Diabetes 2000108 480-485.

12 Sablowski N, Pawlik K, Lüdecke DK \& Herrmann HD. Aspects of personality in patients with pituitary adenomas. Acta Neurochirurgica $1986 \mathbf{8 3} 8-11$.

13 Heald AH, Ghosh S, Bray S, Gibson C, Anderson SG, Buckler H \& Fowler HL. Long-term negative impact on quality of life in patients with successfully treated Cushing's disease. Clinical Endocrinology 200461 458-465.

14 Pikkarainen L, Sane T \& Reunanen A. The survival and well-being of patients treated for Cushing's syndrome. Journal of Internal Medicine 1999245 463-468.

15 Lindholm J, Juul S, Jorgensen JO, Astrup J, Bjerre P, FeldtRasmussen U, Hagen C, Jorgensen J, Kosteljanetz M, Kristensen L, Laurberg P, Schmidt K \& Weeke J. Incidence and late prognosis of Cushing's syndrome: a population-based study. Journal of Clinical Endocrinology and Metabolism 200186 117-123.

16 Johnson MD, Woodburn CJ \& Vance ML. Quality of life in patients with a pituitary adenoma. Pituitary 20036 81-87.

17 Webb SM, Prieto L, Badia X, Albareda M, Catalá M, Gaztambide S, Lucas T, Páramo C, Picó A, Lucas A, Halperin I, Obiols G \& Astorga R. ACROQOL, a new health-related quality of life questionnaire for patients with acromegaly: development and psychometric properties. Clinical Endocrinology 200257 251-258.

18 Badia X, Barahona MJ, Glusman J, Pérez P \& Webb SM. Strategy for developing a specific Cushing's syndrome QoL questionnaire. 12th ISOQOL Conference on Patient Reported Outcomes in Clinical Practice. Budapest (Hungary), 2007 June 24-26 (Abstract).

19 Wagner AK, Gandek B, Aaronson NK, Acquadro C, Alonso J, Apolone G, Bullinger M, Bjorner J, Fukuhara S, Kaasa S, Leplege A, Sullivan M, Wood-Dauphinee S \& Ware JE. Cross-cultural comparisons of the content of SF-36 translations across 10 countries: results from the IQOLA project. Journal of Clinical Epidemiology 199851 925-932.

20 Herdman M, Fox-Rushby J \& Badia X. A model of equivalence in the cultural adaptation of HRQoL instruments: the universalist approach. Quality of Life Research 19987 323-335.

21 Rasch G. Probabilistic Models for Some Intelligence and Attainment Test Chicago. USA: MESA Press, 1980.

22 Grabner P, Hauer-Jensen M, Jervell J \& Flatmark A. Long-term results of treatment of Cushing's disease by adrenalectomy. European Journal of Surgery 1991157 461-464.

23 Biermasz NR, Van Thiel SW, Pereira AM, Hoftijzer HC, Van Hemert AM, Smit JWA, Romijn JA \& Roelfsema F. Decreased quality of life in patients with acromegaly despite long-term cure of growth hormone excess. Journal of Clinical Endocrinology and Metabolism 200489 5369-5376.

24 Gurnell EM, Hunt PJ, Curran SE, Conway CL, Pullenayegum EM, Huppert FA, Compston JE, Herbert J \& Chatterjee VK. A randomised, controlled trial of long-term DHEA replacement in primary adrenal insufficiency. Journal of Clinical Endocrinology and Metabolism 200793 400-409.

25 Hahner S, Loeffler M, Fassnacht M, Weismann D, Koschker AC, Quinkler M, Decker O, Arlt W \& Allolio B. Impaired subjective health status in 256 patients with adrenal insufficiency on standard therapy based on cross-sectional analysis. Journal of Clinical Endocrinology and Metabolism $2007923912-3922$.

26 Bollerslev J, Jansson S, Mollerup CL, Nordenström J, Lundgren E, Tørring O, Varhaug JE, Baranowski M, Aanderud S, Franco C, Freyschuss B, Isaksen GA, Ueland T \& Rosen T. Medical observation, compared with parathyroidectomy, for asymptomatic primary hyperparathyroidism: a prospective, randomized trial. Journal of Clinical Endocrinology and Metabolism 200792 1687-1692.

27 Schroeder PR, Haugen BR, Pacini F, Reiners C, Schlumberger M, Sherman SI, Cooper DS, Schuff KG, Braverman LE, Skarulis MC, Davies TF, Mazzaferri EL, Daniels GH, Ross DS, Luster M, Samuels MH, Weintraub BD, Ridgway EC \& Ladenson PW. A comparison of short-term changes in health-related quality of life in thyroid carcinoma patients undergoing diagnostic evaluation with recombinant human thyrotropin compared with thyroid hormone withdrawal. Journal of Clinical Endocrinology and Metabolism 200691 878-884.

28 Dekkers OM, Biermasz NR, Smit JW, Groot LE, Roelfsema F, Romijn JA \& Pereira AM. Quality of life in treated adult craniopharyngioma patients. European Journal of Endocrinology $2006154483-489$.

29 Razvi S, Ingoe LE, McMillan CV \& Weaver JU. Health status in patients with sub-clinical hypothyroidism. Journal of Clinical Endocrinology and Metabolism 2005152 713-717.

30 Feldt-Rasmussen U, Abs R, Bengtsson BA, Bennmarker $\mathrm{H}$, Bramnert M, Hernberg-Stahl E, Monson JP, Westberg B, Wilton P, Wuster C \& KIMS International Study Board on behalf of KIMS Study Group Growth hormone deficiency and replacement in hypopituitary patients previously treated for acromegaly or Cushing's disease. European Journal of Endocrinology 2002146 67-74.

31 Bourdeau I, Bard C, Noel B, Leclerc I, Cordeau MP, Belair M, Lesage J, Lafontaine L \& Lacroix A. Loss of brain volume in endogenous Cushing's syndrome and its reversibility after correction of hypercortisolism. Journal of Clinical Endocrinology and Metabolism 200287 1949-1954.

32 Barahona MJ, Sucunza N, Farrerons J, Lluch P, Puig T, Wägner AM, van Elzelingen C, Fernández-Real JM \& Webb SM. Increased fat mass in women with 'cured' Cushings syndrome. European Neuroendocrine Association meeting, Athens, Hormones 2006 5 74-75 (Abstract).

Received 23 December 2007

Accepted 21 January 2008 